\title{
Reactions of some annelated 2-aminothiophenes with two naphthoquinones
}

\author{
Emmanuel Sopbué Fondjo $^{a^{*}}$ and Dietrich Döpp ${ }^{b}$ \\ ${ }^{a}$ Laboratory of Applied Synthetic Organic Chemistry,Department of Chemistry, Faculty of \\ Sciences, University of Dschang, P.O.Box 067 Dschang, Republic of Cameroon, \\ ${ }^{b}$ Fachbereich Chemie/Organische Chemie, Universität Duisburg-Essen, D-45117 Essen, Germany, \\ E-mail: *sopbue@yahoo.fr, dietrich.doepp@uni-duisburg-essen.de
}

Dedicated to the memory of Dr. Emmanuel Nyiondi-Bonguen

\begin{abstract}
2-Amino-4,5,6,7-tetrahydrobenzo[b]thiophene-3-carbonitrile (1) and three 3aminobenzopyrano[3,4-c]thiophenes (2a-c) were reacted with 2,3-dichloro-1,4-naphthoquinone (3a) and the parent 1,4-naphthoquinone (3b) in solution at reflux temperature. While from 1 only products of addition/dehydrogenation and chlorine substitution, respectively, were obtained, Diels-Alder addition of $\mathbf{2 a - c}$ to $\mathbf{3 b}$ followed by hydrogen sulfide elimination led to the polycycles 10a-c.
\end{abstract}

Keywords: Anellated 2-aminothiophenes, naphthoquinones, Diels-Alder addition, angular anellation, cyclotrimerization

\section{Introduction}

A detailed examination of the chemical literature shows that in recent years the synthesis of quinonoid natural products has drawn a lot of attention. The application of the Diels-Alder reaction has been so far very useful for this purpose. The consequence is a growing interest in the scope of this reaction. To the best of our knowledge, in contrast to furan ${ }^{1-7}$ and other dienes ${ }^{8-12]}$, very little has been reported on the [4+2]-cycloaddition of quinones and other cycloenones to thiophenes in general and 2-aminothiophenes in particular.

Direct addition of dienophiles in general ${ }^{13-15}$ and quinonoid dienophiles in particular across the butadiene fragment of the thiophene ring system in a [4+2]-mode seems to be rare. Three cases of cycloaddition of 1,4-naphthoquinone to [c]anellated 2-aminothiophenes have been reported recently by Al-Saleh et al. ${ }^{16}$. When these components were refluxed in ethanol, [4+2]cycloaddition across the thiophene ring occurred followed by hydrogen sulfide elimination. 
When, however, the components were subjected to microwave irradiation in the presence of a few drops of acetic acid, the 1,4-dihydroxynaphthalen-2-yl residue was introduced to the hitherto unsubstituted carbon atom $\alpha$ to sulfur in the thiophene $\operatorname{ring}^{16}$. The other successful [4+2]cycloadditions of thiophenes with quinones reported in the literature deal either with reactions in which the sulfur atom in the thiophene reagent is activated through oxidation with peracids ${ }^{5}$, prior to or during the reaction, or with cases in which side chains partly or totally act as diene components. $^{17,18}$

We recently reported ${ }^{19}$ the preparation of the condensed benzoxepin 4 from the thienocoumarin 2a and 2,3-dichloro-1,4-naphthoquinone (3a) in refluxing THF in the presence of triethylamine, as the so far only successful reaction from several attempts to induce reactions between $2 \mathbf{a}$ and numerous quinones.<smiles>N=c1oc2ccccc2c2csc(N)c12</smiles>

2a<smiles>O=C1C(Cl)=C(Cl)C(=O)c2ccccc21</smiles>

3a

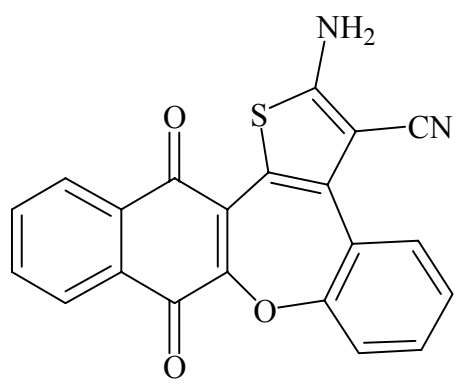

4

\section{Scheme 1}

This discovery prompted us to reexamine in this work some of the reactions of $\mathbf{2 a}$ with quinones under somewhat modified reactions conditions and to extend the study to other 2aminothiophenes such as $\mathbf{1}$ and $\mathbf{2 b , c .}$

\section{Results and Discussion}

Compound 1 reacts with 3a in refluxing toluene in the presence of triethylamine to give in poor yield the aminoquinone 5 (Scheme 2). The latter reaction can be considered as the result of a nucleophilic addition of the amino group of 1a to C-2 of 3a followed by elimination of a molecule of $\mathrm{HCl}$ trapped by triethylamine. It is worth mentioning that in the absence of triethylamine no reaction took place.

By reacting $\mathbf{1}$ with $\mathbf{3 b}$ in boiling dioxane, the aminoquinone $\mathbf{6}$ (Scheme 2) was obtained in low yield after purification by preparative layer chromatography. Structures 5 and $\mathbf{6}$ were assigned on the basis of their IR and mass spectral data.

Reaction of $\mathbf{1}$ with $\mathbf{3 b}$ in refluxing glacial acetic acid gave no addition to the thiophene ring. Besides the $N$-acetylation product 7 , the product $\mathbf{8}$ of the known 
cyclohexadehydrotrimerization $^{20}$ of 1,4-naphthoquinone (3b) was obtained (Scheme 2). This compound crystallizes as a green-yellow, high melting $\left(\mathrm{mp}>360^{\circ} \mathrm{C}\right)$ powder from acetic acid. In the IR spectrum, the absorption bands of the carbonyl groups are seen at $v=1691$ and $1668 \mathrm{~cm}^{-1}$. In the mass spectrum, besides the molecular ion at $\mathrm{m} / \mathrm{z}=468(100 \%)$, characteristic fragmentations are observed at $\mathrm{m} / \mathrm{z}=440(37 \%), 412(49 \%), 356(18 \%), 328(13 \%)$ and 300 (11\%) corresponding to the elimination of $1 \mathrm{x} \mathrm{CO}, 3 \mathrm{x} \mathrm{CO}, 4 \mathrm{x} \mathrm{CO}, 5 \mathrm{x} \mathrm{CO}$ and $6 \mathrm{x} \mathrm{CO}$, respectively.<smiles>N#Cc1c(N)sc2c1CCCC2</smiles>

1<smiles>O=C1C(Cl)=C(Cl)C(=O)c2ccccc21</smiles>

3a
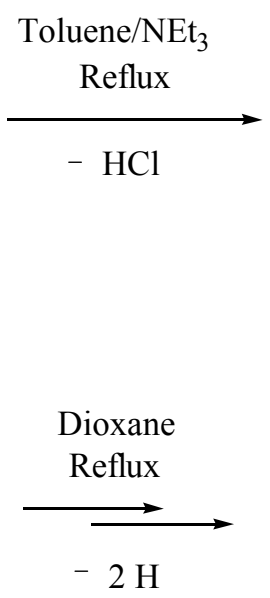

$\mathrm{O}$

$3 b$<smiles>O=C1C=CC(=O)c2ccccc21</smiles><smiles>N#Cc1c(NC2=C(Cl)C(=O)c3ccccc3C2=O)sc2c1CCCC2</smiles>

5<smiles>N#Cc1c(NC2=CC(=O)c3ccccc3C2=O)sc2c1CCCC2</smiles>

6<smiles></smiles>

8

\section{Scheme 2}

According to the literature ${ }^{21}$, the reaction of 1,4-naphthoquinone (3b) with $\mathbf{1}$ cannot be considered as a simple nucleophilic addition, because hydrogen is finally eliminated. From recent kinetic and mechanistic studies ${ }^{22}$, the quinone-amine reactions lead primarily to a chargetransfer-complex intermediate which is subsequently dehydrogenated (either by another molecule of quinone or by oxygen) to final products (aminoquinones) such as 6 (Scheme 2).

The reactions of 2a-c with 1,4-naphthoquinone (3b) in refluxing DMF gave the polycycles 10a-c as the results of Diels-Alder additions across the thiophene rings of 2a-c with subsequent aromatization and $\mathrm{H}_{2} \mathrm{~S}$ elimination (intermediates 9 could not be isolated, Scheme 3). Because of their very poor solubility in DMSO- $\mathrm{d}_{6}$, not all the NMR data were obtained for compounds 10a-

c, the structures of which were nevertheless supported by their elemental analyses, IR and mass spectral data. Furthermore, the more soluble N-trifluoroacetyl derivative 10d, prepared from 10c 
and trifluoroacetic acid anhydride, provided all the analytical and spectroscopic data in agreement with the assigned structures.

Compound 10d crystallizes as an orange powder melting at $274-276{ }^{\circ} \mathrm{C}$ from ethyl acetate. The gross formula $\mathrm{C}_{27} \mathrm{H}_{12} \mathrm{NO}_{5} \mathrm{~F}_{3}$, deduced from the combustion analysis results, was confirmed by the mass spectrum, which exhibited a molecular ion at $\mathrm{m} / \mathrm{z}=487(83 \%)$. The peaks at $\mathrm{m} / \mathrm{z}=$ $418(100 \%)$ and $390(13 \%)$, respectively, were attributed to the ion-fragments $\left(\mathrm{M}^{+}-\mathrm{CF}_{3}\right)^{+},\left(\mathrm{M}^{+}\right.$. $\left.-\mathrm{COCF}_{3}\right)^{+}$. The ${ }^{1} \mathrm{H}-\mathrm{NMR}$ spectrum exhibits in the range $11.93-7.59 \mathrm{ppm}$ a series of signals corresponding to twelve protons. The assignment of ${ }^{1} \mathrm{H}$ - and ${ }^{13} \mathrm{C}\left({ }^{1} \mathrm{H}\right)-\mathrm{NMR}$ data for 10d was done by comparison with those of its precursor 2c and with simulated values as displayed in table 1. The normal ${ }^{13} \mathrm{C}\left({ }^{1} \mathrm{H}\right)$-NMR spectrum contains 27 signals from which 11 signals were assigned to $(\mathrm{C}-\mathrm{H})$-aryl carbon atoms and 16 signals to quaternary $\mathrm{C}$-atoms on the basis of DEPT90/135 experimental data.<smiles></smiles>

2a : $\mathrm{R}^{1}=\mathrm{R}^{2}=\mathrm{H}, \mathrm{X}=\mathrm{NH}$

2b : $\mathrm{R}^{1}=\mathrm{R}^{2}=\mathrm{H}, \mathrm{X}=\mathrm{O}$

2c : $\mathrm{R}^{1}, \mathrm{R}^{2}=-(\mathrm{CH}=\mathrm{CH})_{2^{-}}, \mathrm{X}=\mathrm{O}$<smiles>O=C1C=CC(=O)c2ccccc21</smiles>

$3 b$

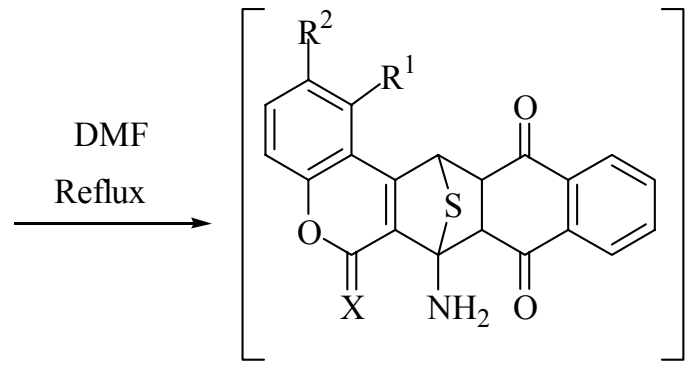

9<smiles>[3H][13CH3]</smiles><smiles></smiles>

10a : $\mathrm{R}^{1}=\mathrm{R}^{2}=\mathrm{H}, \mathrm{R}=\mathrm{H}, \mathrm{X}=\mathrm{NH}$

10b: $\mathrm{R}^{1}=\mathrm{R}^{2}=\mathrm{H}, \mathrm{R}=\mathrm{H}, \mathrm{X}=\mathrm{O}$

(i)

10c: $\mathrm{R}^{1}, \mathrm{R}^{2}=-(\mathrm{CH}=\mathrm{CH})_{2^{-}}, \mathrm{R}=\mathrm{H}, \mathrm{X}=\mathrm{O}$

$\rightarrow \quad 10 d: R^{1}, R^{2}=-(\mathrm{CH}=\mathrm{CH})_{2^{-}}, \mathrm{R}=\mathrm{COCF}_{3}, \mathrm{X}=\mathrm{O}$

(i): $\left(\mathrm{CF}_{3} \mathrm{CO}\right)_{2} \mathrm{O} / \mathrm{CF}_{3} \mathrm{CO}_{2} \mathrm{H}$

\section{Scheme 3}


Table 1. Comparison of ${ }^{1} \mathrm{H}$ - and ${ }^{13} \mathrm{C}\left({ }^{1} \mathrm{H}\right)$-NMR data of $10 \mathrm{~d}$ with the simulated values

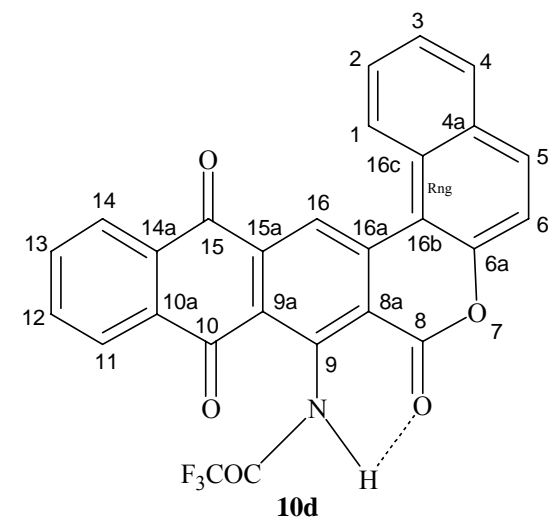

$\delta_{\mathrm{H}}$ in ppm (multiplicity, $\mathrm{J}$ in $\mathrm{Hz}$ )

$\delta_{\mathrm{C}}$ in ppm

\begin{tabular}{|c|c|c|c|c|}
\hline $\begin{array}{c}\mathrm{N}^{\circ} \\
(\mathrm{H}, \mathrm{C})\end{array}$ & Simulated values & Experimental values & $\begin{array}{l}\text { Simulated } \\
\text { values }\end{array}$ & $\begin{array}{c}\text { Experimental } \\
\text { values }\end{array}$ \\
\hline -NH- & $13.143($ br s, $1 \mathrm{H})$ & 11.93 (br s, 1H) & --- & --- \\
\hline 16 & $8.521(\mathrm{~s}, 1 \mathrm{H})$ & $9.24(\mathrm{~s}, 1 \mathrm{H})$ & 114.53 & 116.77 \\
\hline $15 \mathrm{a}$ & --- & --- & 144.64 & 140.16 \\
\hline 15 & --- & --- & 181.78 & 181.32 \\
\hline $14 \mathrm{a}$ & --- & --- & 137.50 & 137.30 \\
\hline 14 & $8.088(\mathrm{dd}, \mathrm{J}=7.61,1.35,1 \mathrm{H})$ & $\begin{array}{c}7.97-7.93(\mathrm{dd}, \mathrm{J}=11.92 \\
2.00,1 \mathrm{H})\end{array}$ & 123.55 & 126.21 \\
\hline 13 & $\begin{array}{c}7.80(\mathrm{ddd}, \mathrm{J}=7.61,7.19,1.35 \\
1 \mathrm{H})\end{array}$ & & 133.60 & 134.62 \\
\hline 12 & $\begin{array}{c}7.825(\mathrm{ddd}, \mathrm{J}=7.61,7.19 \\
1.35,1 \mathrm{H})\end{array}$ & $8.23-8.17(\mathrm{~m}, 2 \mathrm{H})$ & 134.10 & 134.73 \\
\hline 11 & $8.372(\mathrm{dd}, \mathrm{J}=7.61,1.35,1 \mathrm{H})$ & $\begin{array}{c}7.99-7.95(\mathrm{dd}, \mathrm{J}=9.09 \\
1.96,1 \mathrm{H})\end{array}$ & 127.83 & 129.13 \\
\hline $10 \mathrm{a}$ & --- & --- & 136.70 & 137.07 \\
\hline 10 & --- & --- & 186.48 & 181.87 \\
\hline $9 \mathrm{a}$ & --- & --- & 129.36 & 131.43 \\
\hline 9 & --- & --- & 149.06 & 150.80 \\
\hline $8 \mathrm{a}$ & --- & --- & 124.36 & 125.95 \\
\hline 8 & --- & --- & 159.84 & 155.53 \\
\hline $6 a$ & --- & --- & 161.34 & 156.94 \\
\hline 6 & $7.767(\mathrm{~d}, \mathrm{~J}=9.00,0.8,1 \mathrm{H})$ & $8.16-8.13(\mathrm{~d}, \mathrm{~J}=8.03,1 \mathrm{H})$ & 119.45 & 123.79 \\
\hline 5 & $8.094(\mathrm{~d}, \mathrm{~J}=9.00,0.85,1 \mathrm{H})$ & $8.27-8.24(\mathrm{~d}, \mathrm{~J}=9.13,1 \mathrm{H})$ & 135.07 & 135.39 \\
\hline $4 a$ & --- & --- & 133.82 & 132.31 \\
\hline 4 & $7.66(\mathrm{dd}, \mathrm{J}=8.23,1.44,1 \mathrm{H})$ & $7.62-7.59(\mathrm{dd}, \mathrm{J}=8.92$, & 124.49 & 126.78 \\
\hline
\end{tabular}




\begin{tabular}{ccccc} 
& & $1.96,1 \mathrm{H})$ & \\
3 & $7.528(\mathrm{ddd}, \mathrm{J}=8.23,6.96$, & $7.68-7.63(\mathrm{dd}, \mathrm{J}=7.66$, & 125.87 & 127.34 \\
& $1.35,1 \mathrm{H})$ & $7.40,1 \mathrm{H})$ & \\
2 & $7.754(\mathrm{ddd}, \mathrm{J}=8.45,6.96$, & $7.86-7.81(\mathrm{dd}, \mathrm{J}=7.99$, & 122.87 & 124.24 \\
& $1.44,1 \mathrm{H})$ & $7.37,1 \mathrm{H})$ & \\
1 & $8.321(\mathrm{dd}, \mathrm{J}=7.61,1.35,1 \mathrm{H})$ & $8.67-8.65(\mathrm{~d}, \mathrm{~J}=8.64,1 \mathrm{H})$ & 131.97 & 129.89 \\
$16 \mathrm{c}$ & --- & --- & 125.70 & 128.57 \\
$16 \mathrm{~b}$ & --- & --- & 121.31 & 120.79 \\
$16 \mathrm{a}$ & --- & --- & 134.64 & 134.53 \\
$\mathbf{C O C F}_{3}$ & --- & --- & 154.59 & 155.03 \\
$\mathrm{COCF}_{3}$ & --- & -114.25 & 11.33 \\
\hline
\end{tabular}

The reactions of 2-aminothiophenes 2a-c with 1,4-naphthoquinone (3b) under reflux in DMF gave the polycondensed compounds 10a-c and were rationalized in terms of [4+2]cycloaddition followed by $\mathrm{H}_{2} \mathrm{~S}$ release (scheme 3 ). This study has confirmed previous findings ${ }^{23,24}$ on the ability of $[3,4-c]$ benzopyranoanellated 2-aminothiophenes such as 2 a-c to react as electron rich dienes in a [4+2]-mode through their C-3, C-4 bonds, towards electron poor dienophiles such as 1,4-naphthoquinone (3b). Both quinones used in this study are formally electron poor olefins and, at the same time, $\alpha, \beta$-unsaturated carbonyl compounds, oxidants, and electron acceptors. The halogenated quinone 3a is also a vinylene homologous acid chloride. All these properties show up in the encountered results depending on the nature of the reaction and reaction partner.

As previously found ${ }^{24}$, compound $\mathbf{1}$ is not a good diene since establishing a double bond between its $\mathrm{C}-3$ and $\mathrm{C}-3$ a does not profit from a gain in conjugation, the $\mathrm{NH}_{2}$ group thus is nucleophilic and reacts with 2,3-dichloronaphthoquinone as with an acid chloride. If a DielsAlder reaction would occur with $\mathbf{1}$, carbon 7 a would become a quaternary carbon. This result is often difficult to achieve. The salient feature in the Diels-Alder additions of $\mathbf{3 b}$ to $\mathbf{2 a - c}$ is the relation of the polycyclic products $\mathbf{1 0}$ to the class of the important tetracycline and anthracycline antibiotics $^{25}$, which show great promise for the treatment of various tumors and the synthesis of which has exerted a huge influence on the application and development of Diels-Alder chemistry.

\section{Experimental Section}

General Procedures. All the elemental and spectroscopic analyses were performed in the chemistry department analytical center of Gerhard-Mercator-Universität Duisburg, Duisburg (Germany). Melting points were determined with a Reichert Thermovar microscope and are uncorrected. The IR and the UV spectra were measured with Perkin-Elmer 983 and 554 spectrophotometers, respectively. ${ }^{1} \mathrm{H}$ - and ${ }^{13} \mathrm{C}\left({ }^{1} \mathrm{H}\right)$-NMR spectra were recorded on Bruker WM 
300 and DRX 500 instruments, with TMS as internal standard. Coupling constants in brackets are reported in Hertz. Mass spectra were obtained on Varian MAT 311A and AMD 604 instruments by Electron Impact Ionization (EI) at $18 \mathrm{eV}$ or $70 \mathrm{eV}$, using a direct inlet system. Combustion analyses were carried out with a $\mathrm{CHN}+\mathrm{O} / \mathrm{S}$ elemental analyzer "CARLO ERBA" Model 1106. Simulated ${ }^{1} \mathrm{H}$ - and ${ }^{13} \mathrm{C}\left({ }^{1} \mathrm{H}\right)$-NMR-spectra were performed using ACD NMR spectral simulation software.

\section{Starting materials 1a-c and 2}

The starting compounds $2 \mathbf{2}, \mathbf{b},{ }^{26} \mathbf{1},{ }^{27,28}$ were prepared according to described procedures in the yields reported. ${ }^{24}$ Physical, analytical and spectroscopic data are reported for these compounds. The preparation of $\mathbf{2 c}$ was described previously. ${ }^{24,29}$

Reactions of 2-Amino-4,5,6,7-tetrahydrobenzo[b]thiophene-3-carbonitrile (1) 2-[(3-Chloro-1,4-dioxo-1,4-dihydronaphthalen-2-yl)amino]-4,5,6,7-tetrahydrobenzo[b]thiophen-3-carbonitrile (5). A mixture of compound 1 (500 mg, 3 mmol), 2,3-dichloro-1,4naphthoquinone (3a) and triethylamine $(1 \mathrm{~g}, 10 \mathrm{mmol})$ in toluene was heated to reflux under magnetic stirring for $10 \mathrm{~h}$. Concentration in vacuo gave a residue which was taken up in acetone and chromatographed on silica gel plates (cyclohexane $7 /$ ethyl acetate 7.5) to afford $123 \mathrm{mg}$ (11\%) of a crude material, which was crystallized from ethanol to give compound 5 as a red powder, mp $160-162^{\circ} \mathrm{C}$. IR: v/cm ${ }^{-1} 3427,3277(\mathrm{NH}), 2927,2858$ (aryl and aliph. $\mathrm{CH}$ ), 2209 $(\mathrm{CN}), 1670,1635$ ( $\mathrm{C}=\mathrm{O}$ groups). $\mathrm{MS}(\mathrm{EI}): \mathrm{m} / \mathrm{z} 368.0383\left(\mathrm{M}^{+}, 6 \%, \mathrm{C}_{19} \mathrm{H}_{13} \mathrm{ClN}_{2} \mathrm{O}_{2} \mathrm{~S}\right.$ requires 368.0386), 338 (10), 335 (26), 334 (100).

2-[(1,4-Dioxo-1,4-dihydronaphthalen-2-yl)amino]-4,5,6,7-tetrahydrobenzo[b]thiophen-3carbonitrile (6). A mixture of compound 1 (450 $\mathrm{mg}, 2.5 \mathrm{mmol})$ and 1,4-naphthoquinone $3 \mathbf{b}$ $(1.58 \mathrm{~g}, 10 \mathrm{mmol})$ in dioxane was stirred under reflux for $6 \mathrm{~h}$. Concentration in vacuo afforded a crude material, which was dissolved in ethyl acetate and chromatographed on silica gel (hexane 4/ ethyl acetate 1) to give a solid substance, which was crystallized from acetonitrile to give 145 $\mathrm{mg}(17 \%)$ of 6 as a dark-violet powder, mp 228-230 ${ }^{\circ} \mathrm{C}$. IR: $v / \mathrm{cm}^{-1}: 3423,3282(\mathrm{NH}), 2937$ (arom. and aliph. CH), $2209(\mathrm{CN}), 1670,1636(\mathrm{C}=\mathrm{O}), 1611(\mathrm{C}=\mathrm{N}) . \mathrm{MS}(\mathrm{EI}): \mathrm{m} / \mathrm{z} 334.0778\left(\mathrm{M}^{+}\right.$, $100 \%, \mathrm{C}_{19} \mathrm{H}_{14} \mathrm{~N}_{2} \mathrm{O}_{2} \mathrm{~S}$ requires 334.0776), 333 (66), 320 (6), 319 (25), 306 (14), 305 (18), 301 (14), 278 (6), 277 (7), 189 (8), 146 (16), 129 (6), 105 (25).

2-Acetamido-4,5,6,7-tetrahydrobenzo[b]thiophen-3-carbonitrile (7). A magnetically stirred mixture of compound 1 (450 mg, $2.5 \mathrm{mmol}$ ) and 1,4-naphthoquinone (3b, $1.58 \mathrm{~g}, 10 \mathrm{mmol})$ in glacial acetic acid was heated to reflux for $4 \mathrm{~h}$. On cooling to room temperature the precipitate was collected and crystallized from ethanol to give $40 \mathrm{mg}(3 \%)$ of 8 as a green-yellow powder, m.p. $>360^{\circ} \mathrm{C}$ (Lit. ${ }^{[20]}$ : mp was not reported). The resulting filtrate was chromatographed on silica gel (eluent hexane 4/ ethyl acetate 1) to give a crude material which was crystallized from ethyl acetate/cyclohexane to afford $170 \mathrm{mg}(31 \%)$ of 7 as a colorless powder, mp $222-224^{\circ} \mathrm{C}$ (Lit. ${ }^{[28]}$ 216-217 ${ }^{\circ} \mathrm{C}$ from ethanol). IR: $v / \mathrm{cm}^{-1}: 3450,3263,3219(\mathrm{NH}), 3083,3000,2937,2843$ (aliph. C$\mathrm{H}), 2218(\mathrm{CN}), 1692$ (C=O, amide), 1576, 1554, 1463, 1397, 1368, 1347, 1326, 1284, 1255, 1239, 1146, 1034, 997, 953, 856, 821, 765. NMR data: $\delta_{\mathrm{H}}\left(\mathrm{CDCl}_{3}, 300 \mathrm{MHz}\right): 9.27(2 \mathrm{H}$, 
broadened, $\mathrm{NH}), 2.58\left(4 \mathrm{H}, \mathrm{m}, 4-\mathrm{H}_{2}\right.$ and $\left.7-\mathrm{H}_{2}\right), 2.47\left(3 \mathrm{H}, \mathrm{s}, \mathrm{COCH}_{3}\right), 1.80\left(4 \mathrm{H}, \mathrm{m}, 5-\mathrm{H}_{2}\right.$ and 6$\left.\mathrm{H}_{2}\right) . \delta_{\mathrm{C}}\left(\mathrm{CDCl}_{3}, 75 \mathrm{MHz}\right) 167.4(\mathrm{C}=\mathrm{O}), 147.6(\mathrm{C}-2), 130.7(\mathrm{C}-7 \mathrm{a}), 128.1$ (C-3), $114.8(\mathrm{C}-3 \mathrm{a})$, 92.4 (CN), 24.0 (C-7), 23.9 (C-4), 23.1 (C-6), 22.2 (C-5). MS (EI): m/z $222\left(\mathrm{M}^{+}+2,4\right), 221\left(\mathrm{M}^{+}\right.$ $+1,9), 220.0671\left(\mathrm{M}^{+}, 66 \%, \mathrm{C}_{11} \mathrm{H}_{12} \mathrm{~N}_{2} \mathrm{OS}\right.$ requires 220.0670), 219 (2), 180 (5), 179 (12), 178 $\left(\mathrm{M}^{+}-42,100\right), 177\left(\mathrm{M}^{+}-\mathrm{COCH}_{3}, 9\right), 151$ (6), 150 (45). Anal. Calcd. For $\mathrm{C}_{11} \mathrm{H}_{12} \mathrm{~N}_{2} \mathrm{OS}$ : C, 60.00; H, 5.45; N, 12.73; S, 14.55. Found: C, 59.83; H, 5.47; N, 12.58; S, 14.70.

$\mathbf{5 , 6 , 1 1 , 1 2 , 1 7 , 1 8 - H e x a h y d r o t r i - ( 2 , 3 - n a p h t h y l e n ) - 5 , 6 , 1 1 , 1 2 , 1 7 , 1 8 - h e x a o n e ~ ( H e x a d e h y d r o - 1 , 4 - ~}$ naphthoquinone trimer, 8). ${ }^{20} \mathrm{IR}: \mathrm{v} / \mathrm{cm}^{-1} 3445,1691,1668(\mathrm{C}=\mathrm{O}), 1592,1544$. MS (EI): $\mathrm{m} / \mathrm{z}$ $468.0633\left(\mathrm{M}^{+}, 100 \%, \mathrm{C}_{30} \mathrm{H}_{12} \mathrm{O}_{6}\right.$ requires 468.0634), $454\left(\mathrm{M}^{+}-14,8\right), 441(10), 440\left(\mathrm{M}^{+}-\mathrm{C}=\mathrm{O}\right.$, 37), 414 (4), 413 (14), $412\left(\mathrm{M}^{+}-2 \mathrm{x} \mathrm{C}=\mathrm{O}, 49\right), 411$ (5), 385 (8), $384\left(\mathrm{M}^{+}-3 \mathrm{x} \mathrm{C}=\mathrm{O}, 32\right), 383$ (5), 358 (6), $356\left(\mathrm{M}^{+}-4 \mathrm{x} \mathrm{C}=\mathrm{O}, 18\right), 355$ (4), 328 (13), $327\left(\mathrm{M}^{+}-5 \mathrm{x} \mathrm{C}=\mathrm{O}, 14\right), 314$ (4), 300 (11), 299 (12), $298\left(\mathrm{M}^{+}-6 \mathrm{x} \mathrm{C}=\mathrm{O}, 24\right), 297$ (4), 296 (3), 234 (6), 224 (5), 223 (3), 222 (3), 206 (4), 192 (5), 178 (7), 164 (9), 151 (4), 150 (22), 149 (22), 148 (5), 137 (4), 136 (4). Anal. Calcd. for $\mathrm{C}_{30} \mathrm{H}_{12} \mathrm{O}_{6}$ : C, 76.92; H, 2.58. Found: C, 76.29; H, 2.90.

Reactions with 2a,b,c. The reactions of 2a,b,c with 3a in refluxing DMF gave in every case complex mixtures as black powders. The ${ }^{1} \mathrm{H}-\mathrm{NMR}$ and IR of these crude materials gave no reliable structural information.

7-Amino-5-oxa-8,13-dihydro-6-imino-6H-naphtho[1,2-a]phenanthren-8,13-dione (10a). A stirred mixture of compound 2a $(1.08 \mathrm{~g}, 5 \mathrm{mmol})$ and $3 \mathbf{b}(1.58 \mathrm{~g}, 10 \mathrm{mmol})$ was heated to reflux for 7 hours. The solid material was crystallized from DMF/ethyl acetate to give $1645 \mathrm{mg}(97 \%)$ of 10a as a red powder, mp 300-302 ${ }^{\circ} \mathrm{C}$. IR: v/cm ${ }^{-1} 3457,3282\left(=\mathrm{NH}, \mathrm{NH}_{2}\right), 1647(\mathrm{C}=\mathrm{O}), 1590$, $1565,1499,1467,1440,1391,1322,1285,1270,1231,1164,1109,1092,1046,1025$. NMR data: $\delta_{\mathrm{H}}\left(\mathrm{DMSO}_{\mathrm{d}}, 300 \mathrm{MHz}\right)$ and $\delta_{\mathrm{C}}\left(\mathrm{DMSO}_{6} \mathrm{~d}_{6}, 75 \mathrm{MHz}\right)$ the substance was not soluble enough for suitable measurements. MS (EI): $\mathrm{m} / \mathrm{z} 342\left(\mathrm{M}^{+}+2,34\right), 341\left(\mathrm{M}^{+}+1,34\right), 340.0848$ $\left(\mathrm{M}^{+}, 100 \%, \mathrm{C}_{21} \mathrm{H}_{12} \mathrm{~N}_{2} \mathrm{O}_{3}\right.$ requires 340.0848), 339 (14), $324\left(\mathrm{M}^{+}-\mathrm{NH}_{2}, 3\right), 323\left(\mathrm{M}^{+}-\mathrm{NH}_{3}, 8\right)$, 314 (3), $312\left(\mathrm{M}^{+}-\mathrm{CO}, 5\right), 311(11), 296\left(\mathrm{M}^{+}-\mathrm{O}=\mathrm{C}=\mathrm{N}, 4\right), 295$ (10), 294 (5), 256 (3), 228 (3), 227 (4), 170 (6), 129 (4), 114 (5), 106 (4), 105 (6), 101 (4), 100 (4). Anal. Calcd. for $\mathrm{C}_{21} \mathrm{H}_{12} \mathrm{~N}_{2} \mathrm{O}_{3}$ : C, 74.11; H, 3.55; N, 8.23. Found: C, 73.97; H, 3.46; N, 8.58.

7-Amino-5-oxa-8,13-dihydro-6H-naphtho[1,2-a]phenanthren-6,8,13-trione (10b). The same experimental procedure as for 10a was applied, starting from $\mathbf{2 b}(1.09 \mathrm{~g}, 4 \mathrm{mmol})$ and $\mathbf{3 b}(1.58$ $\mathrm{g}, 10 \mathrm{mmol})$ to afford $1686 \mathrm{mg}(98 \%)$ of $\mathbf{1 0 b}$ as red powder, mp $302-304^{\circ} \mathrm{C}$ (from DMF / ethyl acetate). IR: $v / \mathrm{cm}^{-1} 3383,3255\left(\mathrm{NH}_{2}\right), 1707,1669$ (C=O groups), 1602, 1585, 1569, 1527, 1467 , $1441,1389,1363,1327,1289,1249,1212,1161,1108,1072,1051,1030,959,932,868,798$, 754, 710, 617, 545, 467, 420. NMR data: $\delta_{\mathrm{H}}\left(\mathrm{DMSO}_{6}, 300 \mathrm{MHz}\right) 10.09(1 \mathrm{H}$, broadened, NH), $9.22(1 \mathrm{H}$, broadened, NH), $8.21(4 \mathrm{H}, \mathrm{m}$, aryl $\mathrm{H}), 7.92(2 \mathrm{H}, \mathrm{m}$, aryl $-\mathrm{H}), 7.68(1 \mathrm{H}, \mathrm{s}, 14-\mathrm{H}), 7.45$ $\left(2 \mathrm{H}, \mathrm{m}\right.$, aryl-H). $\delta_{\mathrm{C}}\left(\mathrm{DMSO}-\mathrm{d}_{6}, 75 \mathrm{MHz}\right)$ the substance was not soluble enough for suitable measurements. MS (EI): $\mathrm{m} / \mathrm{z} 341.0686\left(\mathrm{M}^{+}, 100 \%, \mathrm{C}_{21} \mathrm{H}_{11} \mathrm{NO}_{4}\right.$ requires 341.0688), $313\left(\mathrm{M}^{+}-\right.$ $\mathrm{CO}, 8)$ and $285\left(\mathrm{M}^{+}-2 \mathrm{x} \mathrm{CO}, 15\right), 257\left(\mathrm{M}^{+}-3 \mathrm{x} \mathrm{CO}, 5\right)$. Anal. Calcd. for $\mathrm{C}_{21} \mathrm{H}_{11} \mathrm{NO}_{4}$ : C, 73.90; H, 3.25; N, 4.10. Found: C, 73.69; H, 2.85; N, 3.50 . 
9-Amino-7-oxa-10,15-dihydro-8H-naphtho[1,2-a]naphthacen-8,10,15-trione (10c). A magnetically stirred mixture of compound $2 \mathbf{c}(935 \mathrm{mg}, 3.5 \mathrm{mmol})$ and $3 \mathbf{b}(1.58 \mathrm{~g}, 10 \mathrm{mmol})$ in DMF was heated to reflux for over $6.5 \mathrm{~h}$ and monitored by TLC. At the end of the reaction, the mixture was kept at room temperature for $24 \mathrm{~h}$. The solid material was collected and crystallized from DMF/ethyl acetate to give $1.34 \mathrm{~g}(98 \%)$ of $10 \mathrm{c}$ as a red powder, $\mathrm{mp} 288-290^{\circ} \mathrm{C}$. IR: $\mathrm{v} / \mathrm{cm}^{-1}$ 3385, $3280\left(\mathrm{NH}_{2}\right), 1704,1668(\mathrm{C}=\mathrm{O}), 1590,1565,1511,1469,1409,1325,1293,1274,1243$, $1217,1160,1083,1048,1029,1007,946,915,814,744,720,695,576,431 . \mathrm{MS}(\mathrm{EI}): \mathrm{m} / \mathrm{z}$ $391.0846\left(\mathrm{M}^{+}, 100 \%, \mathrm{C}_{25} \mathrm{H}_{13} \mathrm{NO}_{4}\right.$ requires 391.0845), 363 (16), 362 (13), 346 (10), 345 (14), 344 (40), 317 (5), 316 (21), 306 (5), 288 (14), 278 (7), 277 (5), 260 (8), 232 (8), 196 (5), 187 (8), 139 (7), 125 (8), 116 (5). Anal. Calcd. for $\mathrm{C}_{25} \mathrm{H}_{13} \mathrm{NO}_{4}$ : C, 76.73; H, 3.32; N, 3.58. Found: C, 76.72; H, 3.38; N, 3.59.

\section{9-Trifluoracetylamino-7-oxa-10,15-dihydro-8H-naphtho[1,2-a]naphthacen-8,10,15-trione} (10d). A mixture of 10c (315 mg) and trifluoroacetic anhydride/trifluoroacetic acid was stirred under reflux for $7 \mathrm{~h}$. The solid material which resulted on cooling was recrystallized from ethyl acetate to afford $207 \mathrm{mg}(53 \%)$ of $\mathbf{1 0 d}$ as an orange powder, mp $274-276^{\circ} \mathrm{C}$. IR: v/ $\mathrm{cm}^{-1} 3454$ $(\mathrm{NH}), 1739,1702,1674$ (C=O groups), 1588, 1537, 1515, 1462, 1446, 1411, 1367, 1307, 1273, $1234,1209,1171,1135,1065,1022,1009,978,910,822,792,747,711,690,607,428$. NMR data: $\delta_{\mathrm{H}}\left(\right.$ DMSO-d $_{6}, 300 \mathrm{MHz}$, see Tab.1). $\delta_{\mathrm{C}}\left(\right.$ DMSO-d $_{6}, 75 \mathrm{MHz}$, see Tab.1). MS (EI): $\mathrm{m} / \mathrm{z}$ $487.0666\left(\mathrm{M}^{+}, 83 \%, \mathrm{C}_{27} \mathrm{H}_{12} \mathrm{NO}_{5} \mathrm{~F}_{3}\right.$ 487.0668), $419(27), 418\left(\mathrm{M}^{+}-\mathrm{CF}_{3}, 100\right), 391\left(\mathrm{M}^{+}+\mathrm{H}-\right.$ $\left.\mathrm{COCF}_{3}, 6\right), 390\left(\mathrm{M}^{+}-\mathrm{COCF}_{3}, 13\right), 291$ (6), 277 (6), 263 (7), 250 (5), 244 (5), 125 (5). Anal. Calcd. for $\mathrm{C}_{27} \mathrm{H}_{12} \mathrm{NO}_{5} \mathrm{~F}_{3}$ : C, 66.53; H, 2.46; N, 2.87. Found: C, 66.67; H, 2.46; N, 2.88 .

\section{Acknowledgements}

The authors wish to thank gratefully the Deutscher Akademischer Austauschdienst (DAAD) for granting Dr. E. Sopbué Fondjo a Ph-D fellowship (grant $N^{\circ}$ A/96/11507). Technical and financial support from Gerhard-Mercator-Universität Duisburg is equally acknowledged. Thanks are also due to the University of Dschang and the Ministry of Higher Education of the Republic of Cameroon for kindly releasing the above named author for the implementation of this work. Generous donation of chemicals by Fonds der Chemischen Industrie is gratefully acknowledged. E.S.F. is indebted to OPCW (Organization for the Prohibition of Chemical Weapons) for sponsoring his participation in the $9^{\text {th }}$ ICCA in Arusha, Tanzania $\left(2^{\text {nd }}-7^{\text {th }}\right.$ August 2004), where these results were presented. 


\section{References and Footnotes}

1. (a) Sasaki, T.; Kanematsu, K.; Iizuka, K.; Izumichi, N. J. Org. Chem. 1976, 41, 1105. (b) Sasaki, T.; Kanematsu, K.; Iizuka, K.; Izumichi, N. Tetrahedron 1976, 32, 2879. (c) Sasaki, T.; Kanematsu, K.; Iizuka, K.; Ando, I. J. Org. Chem. 1976, 41, 1425.

2. (a) Warrener, R. N.; Hammer, B. C. J. Chem. Soc., Chem. Commun., 1981, 942. (b) Warrener, R. N.; Evans, D. A. C.; Russell, R. A. Tetrahedron Lett. 1984, 25, 4833.

3. Makhlouf, M. A.; Rickborn, B. J. Org. Chem. 1981, 46, 2734.

4. (a) Smith, J. G.; Dibble, P. W. J. Org. Chem. 1983, 48, 5361. (b) Smith, J. G.; Welankiwar, S. S.; Shantz, B. S.; Lai, E. H.; Chu, N. G. J. Org. Chem. 1980, 45, 1817.

5. Torsell, K. Acta Chem. Scand. B 1980, 30, 353.

6. Lepage, L.; Lepage, Y. J. Heterocycl. Chem. 1980, 15, 793.

7. Keay, B. A.; Plaumann, H. P.; Rajapaksa, D.; Rodrigo, R. Can. J. Chem. 1983, 61, 1987.

8. Houk, K. N. Acc. Chem. Res. 1980, 13, 361.

9. Gleiter, R.; Paquette, L. A. Acc. Chem. Res. 1983, 16, 328.

10. Brown, F. K.; Houk, K. N. J. Am. Chem. Soc. 1985, 107, 1971.

11. Paquette, L. A.; Kravetz, J. M.; Hsu, L. -Y. J. Am. Chem. Soc. 1985, 107, 1971.

12. (a) Tegmo-Larsson, I.-M.; Roseboom, M. D.; Houk, K. N. Tetrahedron Lett. 1981, 22, 2043. (b) Tegmo-Larsson, I.-M.; Roseboom, M. D.; Rondan, N. G.; Houk, K. N. Tetrahedron Lett. 1981, 22, 2047.

13. Gaertner, R.; Tonkyn, R. G. J. Am. Chem. Soc. 1951, 73, 5872.

14. Clapp, D. B. J. Am. Chem. Soc. 1939, 61, 2733.

15. (a) Kotsuki, H.; Kitagawa, S.; Nishizawa, H.; Kotorayama, T. J. Org. Chem. 1978, 43, 1471.

(b) Kotsuki, H.; Nishizawa, H.; Kitagawa, S.; Ochi, M.; Yamasaki, N.; Matsuoka, K.; Kotorayama, T. Bull. Chem. Soc. Jpn. 1979, 52, 544.

16. Al-Saleh, B.; Abdelkhalik, M. M.; El-Apasery, M. A.; Elnagdi, M- H. J. Chem. Res. 2005, 23.

17. Davies, W.; Porter, Q. N. J. Chem. Soc. 1957, 4958.

18. (a) Latif, N.; Mishriky, N.; Girgis, N. S. J. Chem. Soc., Perkin Trans. 1 1981, 1052. (b) Latif, N. ; Meguid, S. A. Indian J. Chem. 1980, 19B, 975.

19. Nyiondi-Bonguen, E.; Sopbué Fondjo, E.; Tanee Fomum, Z.; Döpp, D. J. Chem. Soc.,Perkin Trans. 1 1994, 2191.

20. (a) Brockmann, H.; Greve, H.; Waldmüller, W. Chem. Ber. 1971, 104, 1436. (b) Brockmann, H. Liebigs Ann. Chem. 1988, 1. (c) Laatsch, H. Liebigs Ann. Chem. 1990, 433. (d) Laatsch, H. Liebigs Ann. Chem. 1985, 605.

21. Thomas Findley K. In Quinones as Synthons In The chemistry of quinonoid compounds, Vol. 2, Part 1, Patai, S.; Rappoport, Z.; John Wiley \& Sons: Chichester, 1988; p 552.

22. Muralikrishna, U.; Krishnamurthy, M. Indian J. Chem. 1983, 22A, 858.

23. (a) Elnagdi, M. H.; Negm, A. M.; Erian, A. W. Liebigs Ann. Chem. 1989, 1255. (b) Elnagdi, M. H.; Erian, A. W. Liebigs Ann. Chem. 1990, 1215. (c) Al-Awadhi, H.; Al-Omran, F.; 
Infantes, L.; Foces-Foces, C.; Jagerovic, M.; Elguero, J.; Elnagdi, M. H. Tetrahedron 1995, 51, 12745. (d) Al-Omran, F.; Khalik, M. M. A.; Al-Awadhi, H.; Elnagdi, M. H. Tetrahedron 52, 1996, 11915. (e) Elghandour, A. H. H.; Hussein, A. H. M.; Elnagdi, M. H.; Harb, A. F. A.; Metwally, S. A. M. J. Prakt. Chem. 1992, 334, 723. (f) Elnagdi, M. H.; Negm, A. M.; Hassan, E. M.; El-Boreiy, A. J. Chem. Res. (S), 1993, 130. (g) Abu-Shanab, F. A.; Wakefield, B.; Al-Omran, F.; Abdel Khalek, M. M.; Elnagdi, M. H. J. Chem. Res. (S) 1995, 488; (M), 1995, 2924.

24. (a) Sopbué Fondjo, E.; Döpp, D.; Henkel, G. Tetrahedron 2006, 62, 7121. (b) Sopbué Fondjo, E. ; Döpp, D. Arkivoc, 2006, (x), 90. (c) See corrigendum on pages 228 and 229 of the same issue.

25. Gunnar, S. Drugs of Natural Origin, A Textbook of Pharmacognosy, $4^{\text {th }}$ Revised Edition, Swedish Pharmaceutical Press, 1999; pp 219.

26. Ried, W.; Nyiondi-Bonguen, E. Liebigs Ann.Chem. 1973, Heft 1, 134.

27. Gewald, K. Chem. Ber, 1965, 98, 3571; Chem. Abstr., 1966, 64, 3451.

28. Manhas, M. S.; Rao, V. V.; Seetheraman, P. A.; Succardi, D.; Pazdera, J. J. Chem. Soc. (C) 1969, 1937.

29. El-Gaby, M. S. A.; Zahran, M. A.; Ismail, M. M. F.; Ammar, Y. A. Il Farmaco, 2000, 55, 227. 